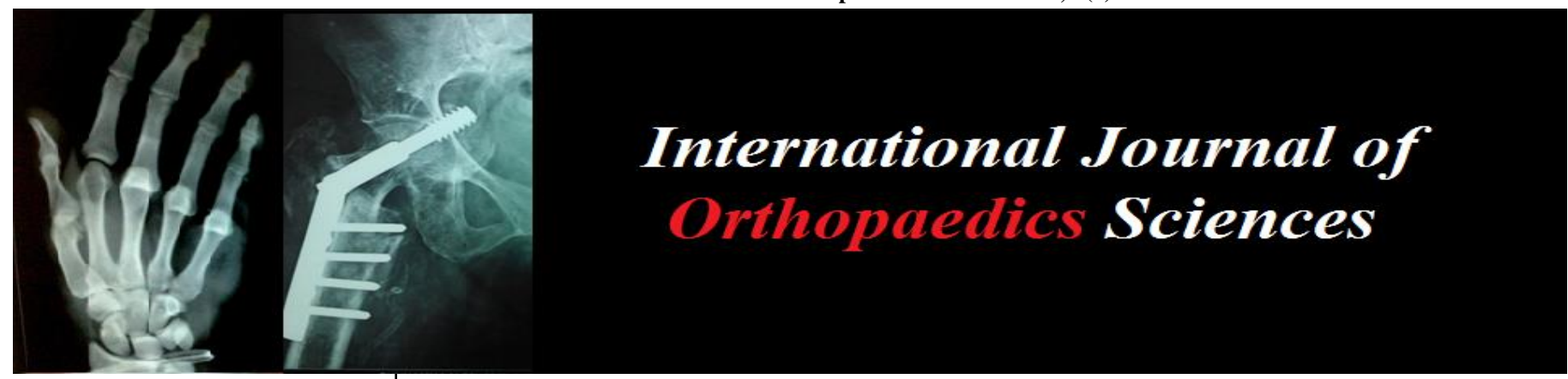

E-ISSN: 2395-1958

P-ISSN: 2706-6630

IJOS 2021; 7(3): 733-736

(C) 2021 IJOS

www.orthopaper.com

Received: 07-05-2021

Accepted: 09-06-2021

\section{Dr. Sanjay Modi}

Associate Professor Department

of Orthopedics, SMIMER

Medical College, Surat, Gujarat,

India

Dr. Nirav Soni

Department of Orthopedics,

SMIMER Medical College, Surat,

Gujarat, India
Corresponding Author: Dr. Sanjay Modi

Associate Professor Department of Orthopedics, SMIMER

Medical College, Surat, Gujarat,

India

\section{Evaluation of results of unstable intertrochanteric femur fracture treated with dynamic hip screw and trochanteric stabilizing plate}

\section{Dr. Sanjay Modi and Dr. Nirav Soni}

DOI: https://doi.org/10.22271/ortho.2021.v7.i3k.2825

\section{Abstract}

Intertrochanteric fractures are more common in the elderly as compared to that in young. These fractures unite readily with conservative line of management as compared to that of neck femur fractures which have an inherent risk of increased complication like avascular necrosis. The study was conducted to evaluate the functional outcome of treating unstable intertrochanteric fractures in individuals using trochanteric stabilising plate [TSP] with dynamic hip screw [DHS]. With prior approval form the ethical committee the study was conducted on the patients admitted in SMIMER, Surat from September 2016 to October 2017 with diagnosed unstable intertrochanteric fracture.

Total 58 patients with unstable intertrochanteric fractures were managed with DHS TSP, of which 48 follow up was possible. Loss of follow up was due to death of 8 patients whereas 2 were due to loss of patient follow up. The patients were informed about the study in all respects and informed written consent was obtained from each for the operative procedure and for enrolment in the study. In the study conclude from that for surgeons familiar with the DHS implant system, the modular TSP extension is a safe effective solution to stabilize selected unstable intertrochanteric fractures.

Keywords: intertrochanteric fracture, dynamic hip screw, trochanteric stabilizing plate

\section{Introduction}

Intertrochanteric fractures are more common in the elderly as compared to that in young [1] These fractures unite readily with conservative line of management ${ }^{[2]}$ as compared to that of neck femur fractures which have an inherent risk of increased complication like avascular necrosis. The unstable intertrochanteric fractures with loss of postero-medial contact or lateral wall contact or both are a major cause of telescoping and medialization with a biomechanically unstable head-neck fragment. This results in significant limb shortening, fracture collapse and implant failure which increase the rates of re-operation.

Parker et al. first documented the importance of integrity of the lateral wall after fixation of these injuries. The management of unstable intertrochanteric fractures is a challenge for orthopaedic surgeons. Accepting this challenge requires a proper understanding of those parameters which determin the outcome ${ }^{[3]}$.

The intact lateral wall plays a key role in stabilisation of unstable trochanteric fractures by providing a lateral buttress for the proximal fragment, and its deficiency leads to excessive collapse and varus malpositioning ${ }^{[3,4]}$ Therefore, maintaining the integrity of this structure should be an important objective in all stabilisation procedures for unstable trochanteric fractures. Moreover, up to $12 \%$ of unstable trochanteric fractures show radiologically identifiable rotation of the proximal fragment, when fixed with DHS alone, as DHS provides only single-point fixation over which the proximal fragment can rotate with the movement of hip. This can result in a significant number of nonunions and malunions due to poor bony contact between two fragments. Trochanter Stabilizing Plate (TSP) is used to stabilize the greater trochanter and the lateral wall and it is used as a modular extension of the Dynamic Hip Screw (DHS). Fixation of unstable intertrochanteric fractures with a TSP have noted to have lesser incidence of femoral medialization and improvement is noted in the functional outcome. 
Here is an effort to study the results of using Trochanteric Stabilising Plate with dynamic hip Screw (DHS) in the management of unstable intertrochanteric fractures.

\section{Aims and Objectives of the Study}

1. To evaluate the functional outcome of treating unstable intertrochanteric fractures in individuals using trochanteric stabilising plate [TSP] with dynamic hip screw [DHS].

2. To evaluate whether the implantation of the modular trochanter stabilizing plate (TSP) in addition to the dynamic hip screw (DHS) prevents medial displacement of shaft (which is frequently associated with the DHS in unstable intertrochanteric fractures)

3. To evaluate whether the implantation of the modular trochanter stabilizing plate (TSP) in addition to the dynamic hip screw (DHS) improve the chances of union and decreases varus collapse with less chances of lag screw cut out

\section{Materials and Methodology}

With prior approval form the ethical committee the study was conducted on the patients admitted in SMIMER, Surat from September 2016 to October 2017 with diagnosed unstable intertrochanteric fracture.

Total 58 patients with unstable intertrochanteric fractures were managed with DHS TSP, of which 48 follow up was possible. Loss of follow up was due to death of 8 patients whereas 2 were due to loss of patient follow up. The patients were informed about the study in all respects and informed written consent was obtained from each for the operative procedure and for enrolment in the study.

\section{Inclusion criteria}

All patients having Unstable intertrochateric fractures, i.e.:

- Evans classification

- AO Classification Type A2.2 and onwards

Willing for surgery \& willing for participation

\section{Exclusion criteria}

High anaesthetic risk

Open and Pathological fractures

Patients with prior surgery, i.e. cases of failed fixation

Any medical contraindication for surgery or underlying major medical conditions hampering outcome like Polio Stroke, Hemiplegia etc.

Stable Intertrochanteric fracture femur, i.e.:

- Evans type 1

- AO Classification Type A1 and A2.1

Patients admitted with intertrochanteric fractures were examined clinically and radiologically.

Above knee skin traction of $5 \mathrm{Kg}$ on Bohler was routinely applied on all cases for pain relief.

Operative Procedure was performed was Closed reduction; sliding hip screw with trochanteric stabilization plate (TSP)

\section{Post-Operative Protocol}

Active SLR and Static Quadriceps strengthening exercises were initiated as soon as patients were out of the Anaesthesia effect. Chest Physiotherapy in the form of deep breathing exercises were started for all patients. Patients were allowed for partial weight bearing, walking with walker from $7^{\text {th }}$ Post operative Day. Full weight bearing was started only after clinical and Radiological signs of union were evident.

\section{Follow Up evaluation}

After fixation of fractures all patients were followed up at 6 weeks 3 months and 6 months and outcome of the ORIF was analysed clinically and radiologically. Clinically the following parameters were assessed and quantified in the form of a score using Modified Harris Hip Score, Range of Motion. And radiologically the parameters used to asses outcome were.

\section{Screw cut out medicalization of shaft Observations}

The average age in the current study came be of 67.2 years which identifies this fracture with this specific classification as a problem of the aged. Also the Age distribution chart shows maximum operated cases with unstable Intertrochanteric fracture femur being of Age $>70$.

$53.2 \%$ patients were female while $46.8 \%$ were female. In 29 $\{56.4 \%\}$ patients, the mechanism of injuey was simple Slip and fall at home- which has been characteristically associated with Osteoporotic cause leading to intertrochanteric femur fracture in the geriatric group. In rest of $43.6 \%$ patients the mode of injury was Road traffic accident majority of those being in the young age group

Range of motion at the end of 6 month post op period was assesed. The range of motion at the hip joint in a normal person is:

The Hip

Flexion

Extension

$5-20$

Abduction

40

Adduction

25

Internal Rotation at 90 Flexion $\quad 45$

External Rotation at 90 Flexion $\quad 45$

Internal Rotation in Extension 35

External Rotation in Extension $\quad 45$

Of the 40 degress of abduction possible $38.3 \%$ of the respondents had 1-20 or more degree of abduction possible. At the end of 6 months follow up $78.7 \%$ of patients had the ability to walk almost similar distance as before operation. 93.6\% of patients were able to wear Shoes, Socks and get ready by themselves for carrying out their day to day affairs when followed up at 6 months post op. The same $93.6 \%$ of patients were able to use public transportation by themselves which included Auto-Rickshwas and local transport buses to get around for carrying out their daily affairs At 6 months follow up $27.7 \%$ of patients had no limp which indicates a functional return with union. $4.2 \%$ had moderate limp but none of them had pain indicating union. $68.1 \%$ had slight limp with most having no to mild pain.

$34 \%$ of patients required no support to walk. One of the benefit of adding TSP to a DHS system is that it prevents medialization of the shaft. In the above study of 48 follow up patients 4 developed medialization of shaft. Out of 35 cases with broken lateral wall there was presence of Medialization of shaft in 4 cases and no medialization in 29 cases.

Table 1: Showing Medialization of Shaft

\begin{tabular}{|c|c|c|}
\hline & Absent & Present \\
\hline $\begin{array}{c}\text { Medialization of Shaft in Lateral wall } \\
\text { Broken Cases }\end{array}$ & 31 & 4 \\
\hline $\begin{array}{c}\text { Medialization of Shaft in Lateral wall Intact } \\
\text { Cases }\end{array}$ & Absent & Present \\
\hline \multicolumn{2}{|c|}{} \\
\hline
\end{tabular}


The average Harris Hip score for patients with Evan's type 3 is 89 , Evan's type 4 is 82.12 whereas for type 5 is 78.35 .

\section{Discussion}

In the present study the average age is 67.2 years. It is comparable to those studies of Indian authors and most of the western authors. This signifies the fact that the patients from these age groups are involved in low energy trauma like: Domestic Fall (Fall at home)

Table 2: Showing Age Distribution of different study

\begin{tabular}{|c|c|c|}
\hline Factor & Current Study & Profet Study [5] \\
\hline Age Distribution (In Years) & 67.2 (On Average) & $60-70$ \\
\hline
\end{tabular}

We had an almost equal Male female incidence with a slight female preponderance in our study, This will be because exposure to trauma amongst the male population is more due to increased risk of day to day exposure to trauma. L.L. Griffin ${ }^{6}$ in their study found a marked female prepondarance in their study $(75.8 \%)$

Table 3: Showing Sex distribution of different study

\begin{tabular}{|c|c|c|}
\hline Factor & Current Study & Griffin Study \\
\hline \multirow{2}{*}{ Sex Distribution } & Female: Male & Female Predominance \\
& $1.16: 1$ & $(75.8 \%)$ \\
\hline
\end{tabular}

The mechanism of injury_was simple slip and fall in $56.4 \%$ of cases and in the rest it was vehicular trauma ${ }^{[7-9]}$. Horn and wang ${ }^{[10]}$ stated that the mechanism of injury is not direct but due to failure of the stress resisting forces during sudden bending or twisting.

Ecker ${ }^{[11]}$ in their study stated that operative line of treatment is the procedure of choice for intertrochanteric fractures The goal of surgical treatment is stable fixation of the fracture. The screw should be placed centrally or inferiorly on anteroposterior view and centrally in lateral view.In our study the screw were placed centrally or inferiorly on ap view and centrally in lateral view. Reduction of the intertrochanteric fracture may be carried out either by open and closed means. In either circumstances the objective is to achieve a stable reduction, be it anatomical or non-anatomical in configuration.

The depth of insertion of the lag screw in to the head is critical for maximum purchase of the proximal fragment. Most agree that it should be inserted within $1 \mathrm{~cm}$ of the subchondral bone, this prnciple has been followed inour series.

Unstable fractures after stabilization only with dynamic hip screw tend to go for varus collapse, telescoping and lateral displocation of greater trochanter, all leading to limb shortening and implant failure.

Table 4: Showing Medialization and Screw cut out of our and other study

\begin{tabular}{|c|c|c|}
\hline Factor & Current Study & Madsen Study $^{[\mathbf{1 2}]}$ \\
\hline Medialisation Of Shaft & $8.3 \%$ & $9 \%$ \\
\hline Screw Cut Out & $8.75 \%$ & $9 \%$ \\
\hline
\end{tabular}

In current study, we saw only $8 \%$ of patients developing medialisation of shaft after placing tsp with dhs in unstable intertrochanteric femur fractures with broken lateral wall. While the rest $92 \%$ patients had good prognosis following tsp with dhs with better Harris hip score too. The medialisation of shaft was much higher for fractures fixed with dhs alone as compared by Madsen. While with lateral wall intact we did not see any medicalization of shaft.

Madsen in his study compared the results after operative treatment of unstable per trochanteric and subtrochanteric fractures with Gamma nail, Compression hip screws or DHS with laterally mounted TSP. In his study all patients were clinically and radiographically assessed three and six months postoperatively. He drew the following results: ${ }^{[13]}$

Use of a TSP reduced the secondary lag screw sliding as compared wit the conventional CHS, without affecting fracture healing. HE concluded that the TSP may be an aid in the treatnebr of these difficult fractures because the problem wit femoral sgaft fractures using the gamma nail is avoided and the medialization of the distal fracture fragment frequently associated with CHS is prevented. In this study the TSP reduced the problem with excessive secondary fracture impaction and medialization of the femoral shaft previously described when using the sliding screw plate systems. Babst et al. found that the lateralization of the greater trochanter was avoided in all cases by using a prototype butress plate with the DHS in 17 patients.

\section{Conclusion}

We conclude from our study that for surgeons familiar with the DHS implant system, the modular TSP extension is a safe effective solution to stabilize selected unstable intertrochanteric fractures.

The complications of immediate weight bearing after fixation of intertrochanteric fractures with only DHS including collapse and medialization of distal fragment leading to shortening and limp can be effectively counteracted by adding TSP to DHS. The combination of DHS with TSP creates a biomechanically stable construct allowing reconstruction of the lateral wall to maintain adequate lever arm and abductor strength. In addition it allows passage of an antirotation screw, thereby providing two point fixation with additional rotational instability. Superior overall functional and radiological outcomes in patients with unstable trochanteric fractures does indicate that a combination of DHS and modular TSP is likely to be a better option in the management of these fractures as compared to DHS alone.

The modular addition of TSP to DHS thus offers a sound solution to the internal fixation of unstable intertrochanteric fractures.

\section{Reference}

1. Alffram PA. An epidemiologic study of cervical and trochanteric fractures of the femur in an urban population analysis of 1,664 cases with special reference to etiologic factors. Acta Orthopaedica Scandinavica 1964;35(65):109.

2. Hornby R, Evans JG, Vardon V. Operative or conservative treatment for trochanteric fractures of the femur. A randomised epidemiological trial in elderly patients. Bone \& Joint Journal 1989;71(4):619-23.

3. Gotfried Y. The lateral trochanteric wall: a key element in the reconstruction of unstable pertrochanteric hip fractures. Clinical orthopaedics and related research. 2004;425:82-6.

4. Fröhlich P, Benkö T. DHS (Dynamic Hip Screw)osteosynthesis in the management of femoral fractures in the hip region and the place of this method in the treatment of such injuries. Magyar traumatologia, ortopedia, kezsebeszet, plasztikai sebeszet 1992;36(1):59-64. 
5. Close J, Ellis M, Hooper R, Glucksman E, Jackson S, Swift C. Prevention of falls in the elderly trial (PROFET): a randomised controlled trial. The Lancet 1999;353(9147):93-7.

6. Griffin JB. The calcar femorale redefined. Clinical orthopaedics and related research 1982;164:211-4.

7. Jensen JS, Tøndevold E, Mossing N. Unstable trochanteric fractures treated with the sliding screw-plate system: a biomechanical study of unstable trochanteric fractures III. Acta Orthopaedica Scandinavica 1978;49(4):392-7.

8. Palm H, Jacobsen S, Sonne-Holm S, Gebuhr P. Hip Fracture Study Group. Integrity of the lateral femoral wall in intertrochanteric hip fractures: an important predictor of a reoperation. JBJS 2007;89(3):470-5.

9. Medoff RJ, Maes K. A new device for the fixation of unstable pertrochanteric fractures of the hip. JBJS 1991;73(8):1192-9.

10. Horn JS, Wang YC. The mechanism, traumatic anatomy, and non-operative treatment of intertrochanteric fracture of the femur. British Journal of Surgery 1964;51(8):57480.

11. Ecker ML, Joyce 3rd JJ, Kohl EJ. The treatment of trochanteric hip fractures using a compression screw. JBJS 1975;57(1):23-7.

12. Madsen JE, Næss L, Aune AK, Alho A, Ekeland A, Strømsøe K. Dynamic hip screw with trochanteric stabilizing plate in the treatment of unstable proximal femoral fractures: a comparative study with the Gamma nail and compression hip screw. Journal of orthopaedic trauma 1998;12(4):241-8.

13. Russel TA. Fractures of hip and pelvis: In Crenshaw AH (Ed): Campbell's Operative Orthopaedics (8th ed). St Louis: CV Mosby 1992, 895. 